Trauma Berufskrankh 2017 · 19 (Suppl 3):S282-S288 https://doi.org/10.1007/s10039-017-0327-x Online publiziert: 17. Oktober 2017

๑) Springer Medizin Verlag GmbH 2017

CrossMark

Die vordere Kreuzbandruptur ist eine der häufigsten Bandverletzungen des Kniegelenks. Der aktuelle Goldstandard der operativen Versorgung ist die Resektion des zerrissenen Kreuzbandes und anschließend die Rekonstruktion mittels autologer Sehne.

In Deutschland wird derzeit v. a. die Semitendinosussehne für diese Sehnenplastik verwendet [7].

Obwohl die Ergebnisse nach VKB (vorderes Kreuzband)-Plastik zu einem Großteil als gut bis sehr gut eingeschätzt werden, gibt es nach der VKB-Plastik nach wie vor Patienten, die weiterhin
M. Herbort · J. Glasbrenner - B. Schliemann - C. Kösters · C. Domnick · M. J. Raschke Klinik für Unfall-, Hand- und Wiederherstellungschirurgie, Universitätsklinikum Münster, Münster, Deutschland

\section{Update vordere Kreuzbandruptur} Ist die Naht eine Option? unter Beschwerden wie einer Rezidivinstabilität oder Schmerzen leiden. Insbesondere die Ergebnisse der Rückführung zum Wettkampfsport und zu pivotierenden Sportarten sind mit gerade einmal $44 \%$ (Rückkehr zum Kontaktsport) als nicht zufriedenstellend einzustufen [5].

Wichtige Nachteile einer konventionellen VKB-Plastik sind weiterhin die Notwendigkeit der Entnahme einer autologen Sehne mit dementsprechend verbundener Entnahmemorbidität, Schwächung von Muskulatur (Semitendinosus - Agonist des VKB und des medialen Kollateralbandes [MCL]) [12], der aktiven medialen Stabilisatoren und Defiziten in der Propriozeption bei Verlust der nervalen und propriozeptiven Potenz des ursprünglichen Kreuzbandgewebes. Weiterhin besteht eine erhöhte Gefahr der Rezidivinstabilität bei ungenauer Platzierung der Bohrkanäle. Diese erschweren im Falle einer Revision das operative Vorgehen und beinhalten häufig die Notwendigkeit eines zweizeitigen Vorgehens mit primärer Tunnelauffüllung mit Spongiosa, bevor eine Revisionsrekonstruktion durchgeführt werden kann.

Die Naht des VKB hingegen ist bereits vor einigen Jahrzehnten durchgeführt worden, wurde jedoch aufgrund von erhöhten Rezidivquoten wieder verlassen [26]. Insbesondere Marshall et al. [19]
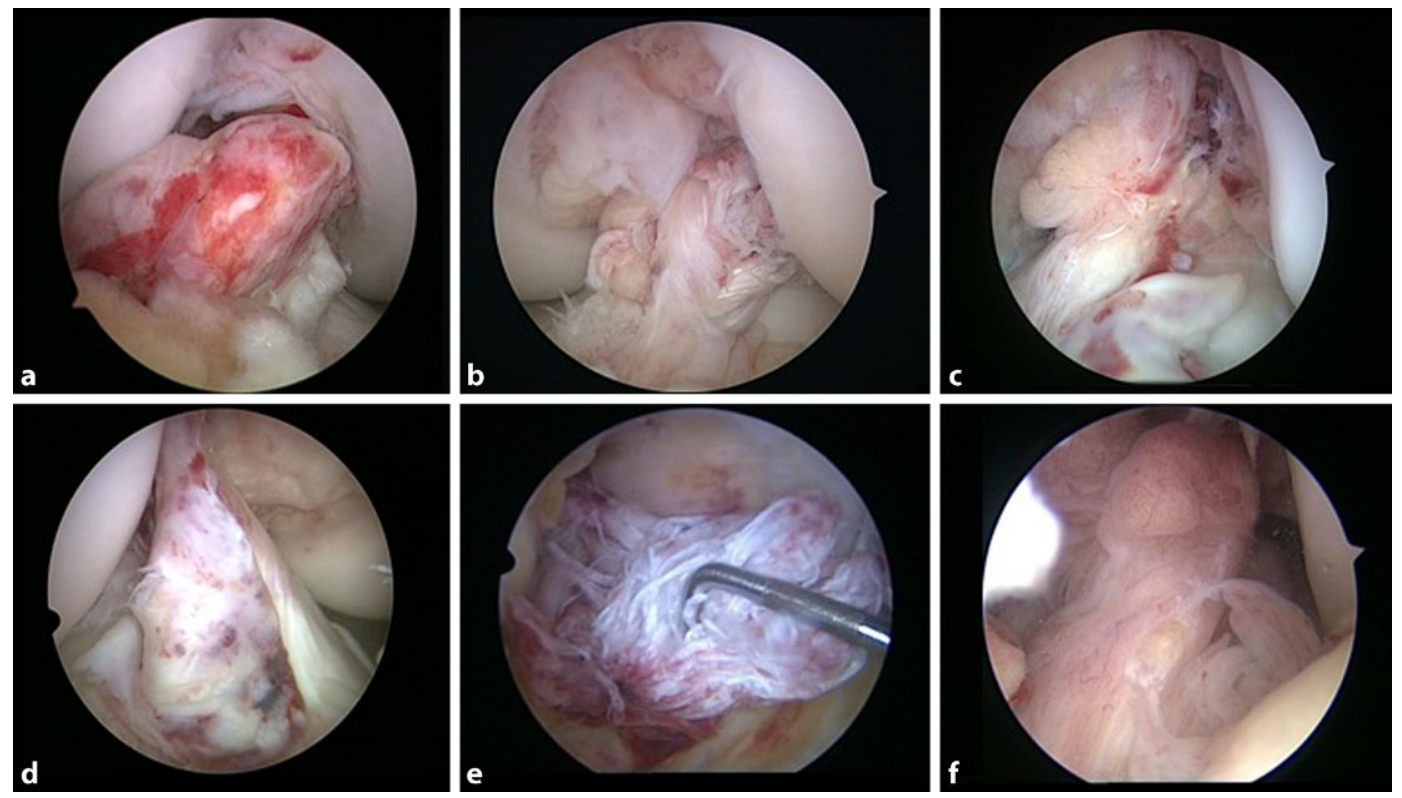

Abb. 1a-f $\Delta$ Unterschiedliche Stumpfkonfigurationen nach Ruptur des vorderen Kreuzbandes. Die Stümpfe unterscheiden sich durch unterschiedliche Rupturhöhe, Integrität des Synovialschlauches und Auffaserung der Bandstrukturen. a proximale VKB Ruptur mit zwei separierten tibialen Stumpfanteilen und teilweise intaktem Synovialschlauch, b intraligamentäre Ruptur mit drei Stumpfanteilen und destruiertem Synovialschlauch, c dreigeteilter tibialer VKB Stumpf mit teilrupturiertem Synovialschlauch, d Zwei-Bündel Ruptur intraligamentär, e stark destruierte intraligamentäre Ruptur mit vollständiger Zerstörung des Synovialschlauches (keine Versorgung mittels Ligamys empfohlen), f Einbündelruptur des VKB mit erhaltenem Synovialschlauch 


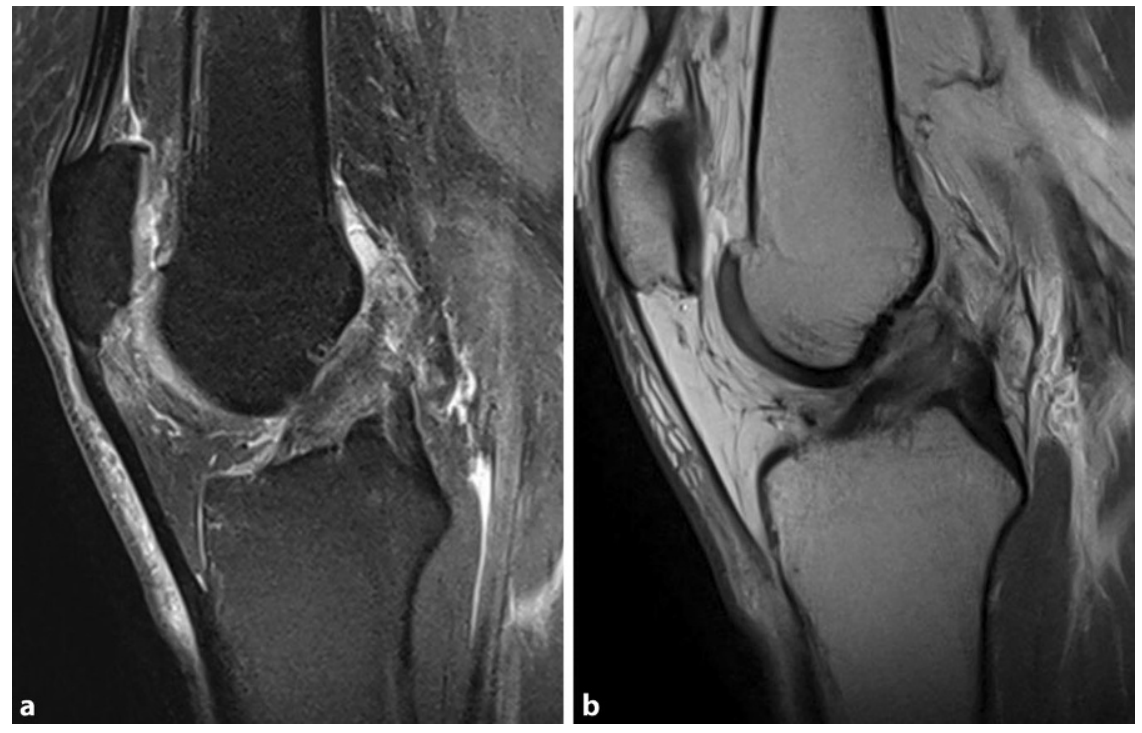

Abb. 2a,b $\Delta$ MRT-Abbildung einer günstigen Stumpfkonfiguration mit noch erhaltenen Anteilen des vorderen Kreuzbandes (VKB) und signalstarken Bandanteilen. Dies ist ein Hinweis auf eine günstige Konfiguration des VKB-Stumpfes ohne starke Auffaserung der Faserstruktur. a PD, b T1
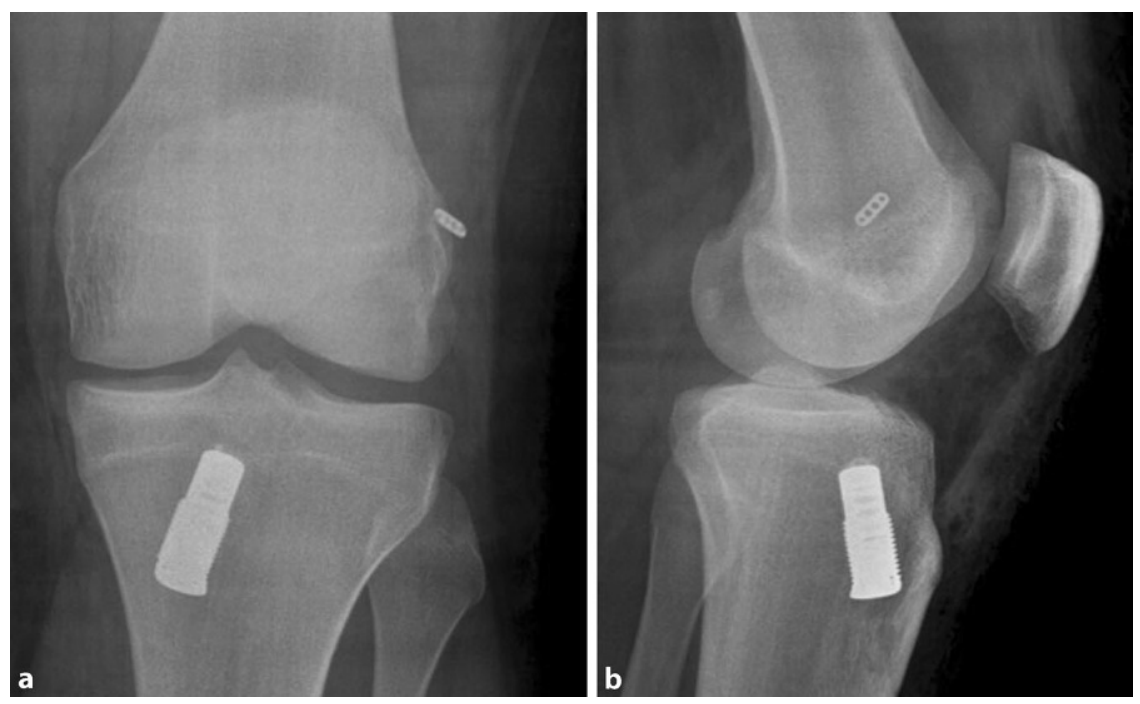

Abb. 3a,b $\boldsymbol{\Delta}$ Röntgenbild in a.-p. (a) und lateralem Strahlengang (b) nach Versorgung mit dem Ligamys ${ }^{\circledR}$-System. Der Abstand des Ligamys ${ }^{\circledR}$-Monoblocks zum Tibiaplateau beträgt $2 \mathrm{~cm}$. Somit ist im Falle einer Reruptur eine einzeitige Revision möglich, ohne den Tunnel aufzufüllen

propagierten direkte Nähte des VKBStumpfes, allerdings mit deutlich reduzierten Ergebnissen. Die Notwendigkeit einer intraartikulären Stabilisierung des Kniegelenks erkannten bereits Paessler et al. [21] und führten diese Stabilisierung mit einem synthetischen Band durch. Nach einem Follow-up von 38 Monaten kehrten jedoch nur $51 \%$ der Patienten auf ihr vorheriges Sportniveau zurück.

Insbesondere durch die Neuentwicklung des Ligamys ${ }^{\circledR}$-Systems (Fa. Mathys)

\section{Indikation zur vorderen Kreuzband-erhaltenden Operation}

Eine wichtige Voraussetzung für die Durchführung einer banderhaltenden Operation ist sicherlich das Alter der Ruptur. Obwohl es keine evidenzbasierten Daten hierzu gibt, ist davon auszugehen, dass eine banderhaltende Operation innerhalb von 2 bis 3 Wochen durchgeführt werden sollte. Bei älteren Rupturen ist v. a. eine Verkürzung und Abrundung des Bandstumpfes zu sehen, die eine Readaption des Stumpfes zum Femur und eine primäre Einheilung unwahrscheinlich machen. Weiterhin scheinen die Höhe der Ruptur und die Konfiguration des Stumpfes eine Rolle zu spielen. Je weiter proximal die Ruptur gelegen und unversehrter der tibiale Stumpf (Anzahl der separierten Bündel, Erhalt des Synovialschlauches) ist, desto günstiger scheinen die Voraussetzungen für eine erhaltende Operation zu sein (• Abb. 1). Evidenzbasierte Daten, die diese empirische Einschätzung untermauern, sind jedoch noch nicht publiziert.

Wichtige Voraussetzungen für die Durchführung eines operativen Banderhaltes sind somit die frühzeitige Durchführung einer Magnetresonanztomographie (MRT) und die genaue Analyse der Rupturform. Van der List et al. [16] konnten in einer aktuellen Studien zeigen, dass mithilfe einer konventionellen MRT sowohl die Rupturhöhe als auch Qualität des Stumpfes in vielen Fällen zuverlässig vorausgesagt werden konnten (• Abb. 2).

Indikationen zur banderhaltenden VKB-Operation sind:

- Operation innerhalb von 2 bis 3 Wochen (Ruptur nicht älter als 3 Wochen),

- möglichst proximale Ruptur,

- möglichst intakter tibialer VKBStumpf.

\section{Dynamische intraligamentäre Stabilisierung mittels Ligamys ${ }^{\circledR}$-System}

Der entscheidende Unterschied zwischen dem Ligamys ${ }^{\circledR}$-System und der 
vorherigen VKB-Naht und Augmentationstechniken ist die dynamische Stabilisierung. Diese orientiert sich an den Erkenntnissen von Lubowitz [18], die zeigen konnten, dass sich die Länge eines anatomisch platzierten VKBTransplantates beim Bewegen des Kniegelenks von $0^{\circ}$ Extension bis $120^{\circ}$ Flexion um durchschnittlich $6,7 \mathrm{~mm}$ ändert. Die Fixation der Ligamys ${ }^{\circledR}$-Kordel mit dem Federsystem erlaubt eine $8 \mathrm{~mm}$-Exkursion und somit eine kontinuierliche Spannung im gesamten Bewegungsausmaß des Kniegelenks. Auf diese Weise wird die Adaption der Bandstümpfe gewährleistet und der störende Einfluss von Zug- und Scherkräften minimiert. Die Indikation zur Versorgung mittels Ligamys ${ }^{\circledR}$-Operation entspricht grundsätzlich den allgemeinen Indikationen aller VKB-erhaltender Operationstechniken.

Beim Ligamys ${ }^{\circledR}$-System gibt es noch spezifische Kontraindikationen:

- Unverträglichkeit gegen die Baustoffe des Systems (z. B. Kobalt, Chrom und Nickel),

- offene Wachstumsfugen (derzeit keine ausreichende Studienlage vorhanden).

Die wichtigsten operativen Schritte des Verfahrens sind im Folgenden dargestellt:

- Mithilfe eines tibialen Zielgeräts Einbringen eines 2,3 mm-K-Drahtes unmittelbar dorsal des tibialen VKBStumpfes. Dieser Draht wird mit einem $10 \mathrm{~mm}$-Bohrer bis auf eine Tiefe von $30 \mathrm{~mm}$ überbohrt, um anschließend die Implantation des Ligamys ${ }^{\circledR}$ Monoblocks zu ermöglichen, in dem sich der Federmechanismus befindet. Der K-Draht sollte mindestens mit einem $60 \mathrm{~mm}$-Abstand zum Tibiaplateau eingebracht werden, um den Monoblock mindestens $2-3 \mathrm{~cm}$ unterhalb des Tibiaplateaus zu verankern (• Abb. 3). Dies ermöglicht im Falle einer Reruptur die problemlose einzeitige Revision mittels VKBPlastik.

- Naht des VKB-Stumpfes mittels einer speziellen Nahtzange von 4 bis 5 PDS (Polydioxanon) 2.0-Fäden, die aus dem anteromedialen Portal ausgleitet werden (• Abb. 4a).

Trauma Berufskrankh 2017 · 19 (Suppl 3):S282-S288

https://doi.org/10.1007/s10039-017-0327-x

(c) Springer Medizin Verlag GmbH 2017

\section{Herbort · J. Glasbrenner · B. Schliemann · C. Kösters · C. Domnick · M. J. Raschke Update vordere Kreuzbandruptur. Ist die Naht eine Option?}

\section{Zusammenfassung}

Fragestellung. Diese Arbeit gibt einen Überblick über den aktuellen Stand der Entwicklung von erhaltenden Operationen des vorderen Kreuzbandes (VKB) und deren klinischen Ergebnissen.

Ergebnisse. In den letzten 5 Jahren hat die VKB-erhaltende Operation eine neue Bedeutung bekommen, und neue Operationstechniken versprechen verbesserte Ergebnisse. Während refixierende Operationstechniken neue Ankertechniken und neu entwickeltes Fadenmaterial verwenden, bieten neue Augmentationstechniken eine Erweiterung des Spektrums und neue Behandlungsperspektiven. Die refixierenden scheinen - bei geringer Datenlage - nach 28 Monaten eine relativ hohe Reoperationsquote von $20 \%$ aufzuweisen. Auch über die nichtdynamische Augmentation mittels Ligament-Bracing bei isolierten VKBRupturen mit einem FiberTape (Arthrex, Naples, USA) existieren keine mittelfristigen klinischen Daten. Ein 1 Jahres-Follow-up wies eine Reoperationsquote von 4 von 68 Patienten auf. Die beste Studienlage besteht bezüglich der dynamischen intraligamentären Stabilisierung mittels des Ligamys ${ }^{\circledR}$-Systems (Mathys AG, Bettlach, Schweiz). Studien berichten über klinische Verlaufsergebnisse von 2 bis 5 Jahren. Hier scheinen vergleichbare Scoreergebnisse wie nach VKB-Rekonstruktion - jedoch mit leicht erhöhten Reruptur- und Insuffizienzquoten - vorzuliegen. Hinsichtlich der biologischen Heilungsproblematik des intraartikulär liegenden VKB gibt es erste Lösungsansätze mittels Kollagenmatrizes, die in experimentellen tierischen Studien mit vielversprechenden Ergebnissen angewandt wurden.

Schlussfolgerungen. Der Erhalt des VKB scheint operativ möglich zu sein. Das Ligamys ${ }^{\circledR}$-Verfahren scheint eine suffiziente Stabilisierung des Kniegelenks zu erreichen. Eine biologische Unterstützung der Heilung ist sinnvoll und klinisch notwendig, jedoch erst im experimentellen Stadium.

Schlüsselwörter

Operation - Refixation - Augmentation . Kollagenmatrizes · Gelenkstabilisierung

\section{Update on anterior cruciate ligament rupture. Is suturing an option?}

Abstract

Objective. This article gives an overview of the current state of development of operations for preservation of the anterior cruciate ligament $(A C L)$ and the clinical results.

Results. In the last 5 years $A C L$ preserving operations have gained new importance and various new surgical techniques promise improved results. While operative techniques for refixation use new anchoring techniques and newly developed suture material, new augmentation techniques provide an extension of the spectrum and new treatment perspectives. Refixation techniques seem to have a relatively high reoperation rate of $20 \%$ after 28 months but few data are available; however, no medium term data are available even for non-dynamic augmentation using ligament bracing with a FiberTape (Arthrex) for isolated ACL ruptures. A 1-year followup showed a reoperation rate of 4 out of 68 patients. The best investigated technique is dynamic intraligamentary stabilization using the Ligamys ${ }^{\circledR}$ system. Several studies reported clinical follow-up results of 2-5 years and comparable score results to $\mathrm{ACL}$ reconstruction but with slightly increased rerupture and insufficiency rates. With respect to the biological healing problem of intraarticular ACLs, there are first approaches to solutions using collagen matrices, which have been applied in experimental animal studies with promising results.

Conclusion. Preservation of the ACL appears to be surgically possible. The Ligamys ${ }^{\circledR}$ method seems to achieve sufficient stabilization of the knee joint. Biological support of the healing appears clinically necessary but is only in the experimental stage.

\section{Keywords}

Operative procedures - Refixation . Augmentation · Collagen matrix $\cdot$ Joint stabilization 

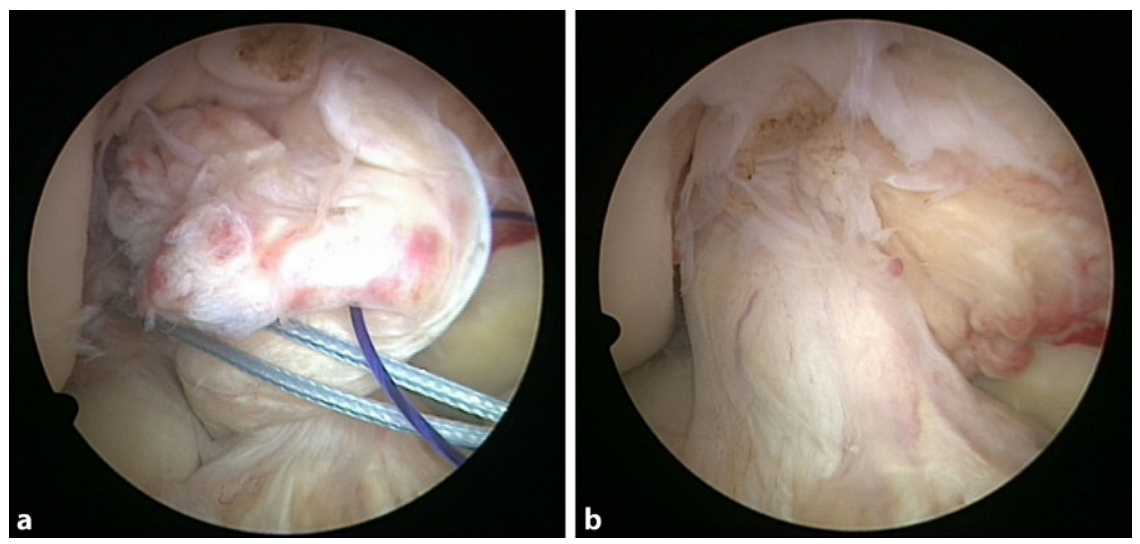

Abb. $4 \Delta$ a VKB (vorderes Kreuzband)-Stumpf mit einem PDS(Polydioxanon)-Faden angeschlungen. Die FibreWire-Durchzugsschlaufe (FibreWire, Arthrex, Naples, USA) zur femoralen Ausleitung der PDSFäden wurde bereits vorgelegt. b VKB-Stumpf nach vollständiger Annaht mit 4 PDS-Fäden und Reposition mittels der PDS-Fäden an die femorale Insertion

- Einbringen eines 2,3 mm-K-Drahtes mit Öse in den femoralen VKBInsertionsbereich durch ein anteromediales Portal. Mithilfe dieses Ösendrahtes werden 2 Durchzugsschlaufen vorgelegt. Mit der ersten Schlaufe wird die Ligamys ${ }^{\circledR}$-Kordel retrograd vom lateralen Oberschenkel durch das Kniegelenk aus dem Monoblock herausgezogen, sodass der Endobutton am lateralen Kortex zu liegen kommt und das freie Ende der Kordel aus dem Monoblock ausgeleitet wird. Mit der zweiten Durchzugsschlaufe werden die 4 bis 5 PDS-Nähte durch dasselbe Loch von intraartikulär durch die laterale Kondyle ausgeleitet.

- Durch das Anziehen der PDS-Nähte wird der VKB-Stumpf zur lateralen Notchwand reponiert und anschließend die PE(Polyethylen)-Kordel bis zum Anliegen des Endobuttons am lateralen Kortex angezogen (-Abb. 4b).

- Die PDS-Fäden werden durch die sich verdickende PE-Kordel in dem gemeinsamen 2,3 mm-Tunnel durch das Anziehen der Kordel verblockt.

- Zur Anregung der Heilung und Anreicherung des intraartikulären Milieus mit Stammzellen erfolgt eine Mikrofrakturierung der Notch.

- Mithilfe des Tensiometers wird das Ligamys ${ }^{\circledR}$-Federsystem mit
60-80 N (je nach Körpergewicht des Patienten) in Extensionsstellung des Kniegelenks vorgespannt.

\section{Nachbehandlung nach Ligamys ${ }^{\circledR}$ Operation}

Bei isolierten VKB-Rupturen erfolgt die Nachbehandlung nach dem folgenden Schema:

- 4 Tage Mecron-Schiene und $20 \mathrm{~kg}$ Teilbelastung an Unterarmgehstützen;

- anschließend Vollbelastung ohne Benutzung von Unterarmgehstützen;

- intensive Beübung des freien Bewegungsumfanges, um v. a. ein Extensionsdefizit zu verhindern;

- ab der 4. Woche dürfen bereits vorsichtige Kraftübungen in der geschlossenen Kette durchgeführt werden;

- eine Rückkehr zum Sport sollte jedoch auch bei diesem Verfahren erst nach einem erfolgreichen Backto-Sports-Test erfolgen. Eine Rückkehr in Level-1-Sportarten wird vor 9 Monaten jedoch als sehr unwahrscheinlich angesehen.

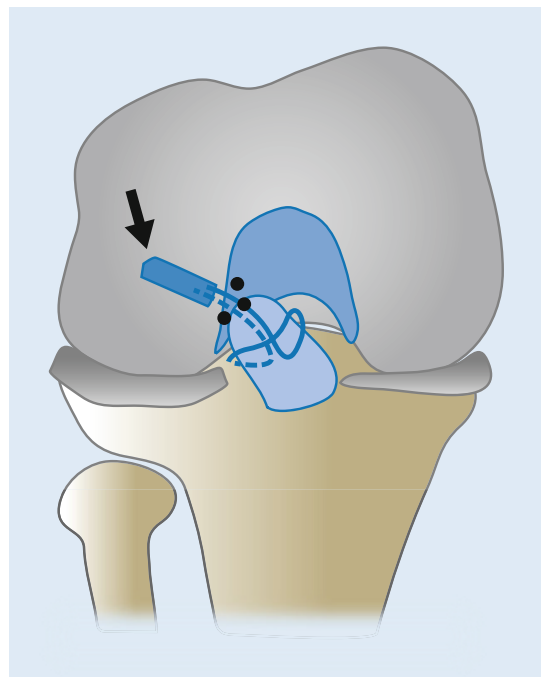

Abb. 5 ॥ Schematische Abbildung einer Ankerrefixationstechnik des tibialen vorderen Kreuzbandstumpfes. Pfeil Druckschloss, schwarze Punkte Mikrofrakturierungslöcher

\section{Aktuelle experimentelle und biomechanische Studienlage des Ligamys ${ }^{\circledR}$-Systems}

Die biomechanische Wirksamkeit von unterschiedlichen Augmentationstechniken wurde bereits mehrfach in $\mathrm{Ka}$ daverstudien untersucht. Mehrere Arbeitsgruppen konnten unabhängig in ihren Untersuchungen eine erfolgreiche Wiederherstellung der physiologischen Gelenkkinematik des Kniegelenks durch das Ligamys ${ }^{\circledR}$-System zeigen $[9,13,22]$. Hoogeslag et al. [13] haben alle gängigen Techniken (Marshall-Naht, LigamentBracing mit FiberTape und Ligamys ${ }^{\circledR}$ ) bezüglich der Wiederherstellung der physiologischen Gelenkkinematik untersucht. Dabei konnte allein das Ligamys ${ }^{\circledR}$ System die Gelenkkinematik im Kadavermodell wiederherstellen.

\section{Aktuelle klinische Studienlage und Ergebnisse nach Ligamys ${ }^{\circledR}$ Operation}

Es sind mittlerweile mehrere Fall-Kontroll-Studien von Patienten nach Versorgung mit der Ligamys ${ }^{\circledR}$-Technik publiziert worden. Die $\bullet$ Tab. 1 fasst diese Publikationen zusammen.

In der bisher einzigen randomisierten, kontrollierten klinischen Studie konnten durch die Autoren jeweils 30 Patienten in den ersten 3 Wochen nach VKB- 
Tab. 1 Auflistung der derzeit publizierten Studien über die klinischen Ergebnisse nach Versorgung mit dem Ligamys ${ }^{\circledR}$-System

\begin{tabular}{llllllll}
\hline Studie & N & $\begin{array}{l}\text { FU } \\
\text { Monate }\end{array}$ & $\begin{array}{l}\text { VAS } \\
(\mathbf{1 - 1 0 )})\end{array}$ & $\begin{array}{l}\text { Lysholm- } \\
\text { Score } \\
(\mathbf{1 - 1 0 0 )}\end{array}$ & $\begin{array}{l}\text { IKDC } \\
(\mathbf{1 0 0} \%)\end{array}$ & Tegner & $\begin{array}{l}\text { Lachman vs. } \\
\text { kontralateral }\end{array}$ \\
\hline Henle [11] & 216 & 12 & 8,9 & 96,07 & 93,5 & 5,1 & $1,4 \mathrm{~mm}$ \\
& 62 & 24 & 9 & 97,4 & 95,4 & 5,1 & $1,9 \mathrm{~mm}$ \\
\hline Kösters [15] & 55 & 12 & 8 & 91,6 & 88,3 & 4,8 & $1,7 \mathrm{~mm}$ \\
\hline Eggli [8] & 10 & 24 & 9,8 & 100 & 98 & 6 & $1,4 \mathrm{~mm}$ \\
\hline $\begin{array}{l}\text { Schliemann } \\
\text { [24] }\end{array}$ & 176 & 12 & 8,9 & 96,07 & 8 & 5,1 & $1,3 \mathrm{~mm}$ \\
\hline
\end{tabular}

[24]

FU "Follow up", VAS visuelle Analogskala Schmerz, IKDC "International Knee Documentation Comittee Score"

Ruptur mit dynamischer intraligamentärer Stabilisierung (DIS) im Ligamys ${ }^{\circledR}$ Verfahren oder mittels 4-fach Semitendinosus-Sehnentransplantat in Hybridfixierung (femoral Endobutton, tibial Interferenzschraube und Endotack, Fa. Karl Storz, Tuttlingen, Deutschland) versorgt werden.

Beide Gruppen wurden nach dem üblichen Ligamys ${ }^{\circledR}$-Schema nachbehandelt (s. Abschn. „Nachbehandlung nach Ligamys ${ }^{\circledR}$-Operation“) Als Zielparameter wurden Tegner-, Lysholm- und IKDC(„International Knee Documentation Comittee Score“)-Score sowie eine funktionelle klinische Untersuchung und eine Ganganalyse mit Schrittzähler erhoben.

In der laufenden Erhebung der 2-Jahres-Ergebnisse liegt die Rerupturrate bei $3 / 27$ nach DIS sowie bei 3/25 nach VKBPlastik. Die Rate der Reoperationen liegt bei 7/27 in den ersten 2 Jahren nach DIS im Vergleich zu 2/25 nach VKB-Plastik. Als Operationsindikation wurden intraartikuläre Vernarbungen mit Bewegungseinschränkungen oder implantatassoziierten Beschwerden im Bereich des tibialen Monoblocks angegeben.

Die anteriore Translation im Rolimeter-Lachman-Test konnte durch DIS nach $1 \mathrm{Jahr}$ von 7,6 $\mathrm{mm}$ auf $1,7 \mathrm{~mm}$ sowie durch die VKB-Plastik von $8,4 \mathrm{~mm}$ auf $1,4 \mathrm{~mm}$ reduziert werden. Der numerische Vorteil der VKB-Plastik war hierbei nicht signifikant und auch der Vergleich des Tegner-, Lysholm- und IKDC-Scores erbrachte vergleichbare Ergebnisse.

In der Schrittzählermessung konnte nach 6 Wochen eine signifikant höhere Aktivität der DIS-Gruppe nachgewie- sen werden, was im Sinne einer früheren beruflichen Rehabilitation durchaus Relevanz haben kann. In den weiteren Follow-ups nach 6 Monaten und 1 Jahr nivellierten sich die Unterschiede in der Aktivitätsmessung [23].

Die vom Hersteller im Nachbehandlungsschema angegebene Return-toSports-Zeit ab dem 5. postoperativen Monat kann nach eigener klinischer Erfahrung - auch bei entsprechendem muskulärem Status - nicht empfohlen werden. Aus Sicht der Autoren besteht auch nach banderhaltender Operation bei einer Rückkehr in pivotierende Sportarten vor 10 bis 12 Monaten postoperativ ein deutlich erhöhtes Rerupturrisiko.

Zusammenfassend zeigt die bisher einzige randomisierte, kontrollierte klinische Studie einer banderhaltenden, operativen Versorgung nach VKBRuptur in einer Zwischenauswertung der 2-Jahres-Nachuntersuchungen vergleichbare klinische Ergebnisse zur VKBPlastik mit autologem Sehnentransplantat. Allerdings bedingen intraartikuläre Vernarbungen sowie implantatassoziierte Beschwerden eine erhöhte Reoperationsrate nach DIS.

\section{Ankerrefixationstechniken beim vorderen Kreuzband ohne Augmentation}

Neben der Ligamys ${ }^{\circledR}$-Technik sind in den letzten Jahren Ankerrefixationstechniken zur Reinserierung und Refixation von tibialen VKB-Stümpfen weiterentwickelt und deren kurzfristige Ergebnisse publiziert worden [17]. Die Refixation erfolgte hierbei mit modernen kleinen
Ankersystemen im Footprint des femoralen VKB-Ursprungs [1, 2, 6]. Mit diesen Verfahren wurden v. a. proximale VKB-Rupturen mit qualitativ hochwertigem tibialem Stumpf ohne zusätzliche Augmentation bzw. innerer Schienung des Kniegelenks refixiert [1, 2, 6]. Bezüglich der biomechanischen Stabilität dieser Fixationstechniken gibt es leider nur eine nicht aussagekräftige biomechanische Arbeit von van der List et al., die im humanen Kadaverkniemodell eine zyklische Extensions-Flexions-Bewegung mit 100 Zyklen durchführten und Ausreißversuche mit einer mittleren maximalen Ausreißkraft von $243 \mathrm{~N}$ (zwischen $65 \mathrm{~N}$ und $299 \mathrm{~N}$ ) durchführten. Diese Daten geben leider nur wenig valide Informationen über die Stabilität und Sicherheit dieses Verfahrens in der klinischen Anwendung.

Zwei Arbeitsgruppen haben jedoch mittlerweile ihre kurzfristigen klinischen Ergebnisse bezüglich Ankerrefixation von VKB-Rupturen publiziert. So berichteten Achtnich et al. [1] in einer FallKontroll-Studie über 20 Patienten, die mit einer Nahtankerrefixation versorgt und mit 20 Patienten nach VKB-Rekonstruktion verglichen wurden (• Abb. 5). Während in der Rekonstruktionsgruppe nach einem Follow-up von 28 Monaten kein Therapieversager und keine Revisionsoperation ermittelt wurden, wurden in der Ankerrefixationsgruppe eine Versagerquote von $15 \%$ und eine Reoperationsquote von $20 \%$ festgestellt [1]. Die im Rahmen der Studie ermittelten Scores zeigten zwischen den beiden Gruppen keinen signifikanten Unterschied.

DiFelice et al. [6] berichten ebenfalls über kurzfristige Ergebnisse mit einem Mindestverlaufszeitpunkt von 2 Jahren. Die Refixation von femoralen VKB-Rupturen erfolgte in diesem Kollektiv mittels FibreWire/TigerWire (Arthrex, Naples, USA) und 2 4,75 mm Bio-Composite-SwiveLock-Ankern (Arthrex, Naples, USA). In dem Kollektiv zeigten 10 von 11 Patienten ein gutes subjektives und klinisches Outcome und 7 von 8 Patienten eine anteriore tibiale Translation von weniger als $3 \mathrm{~mm}$. 

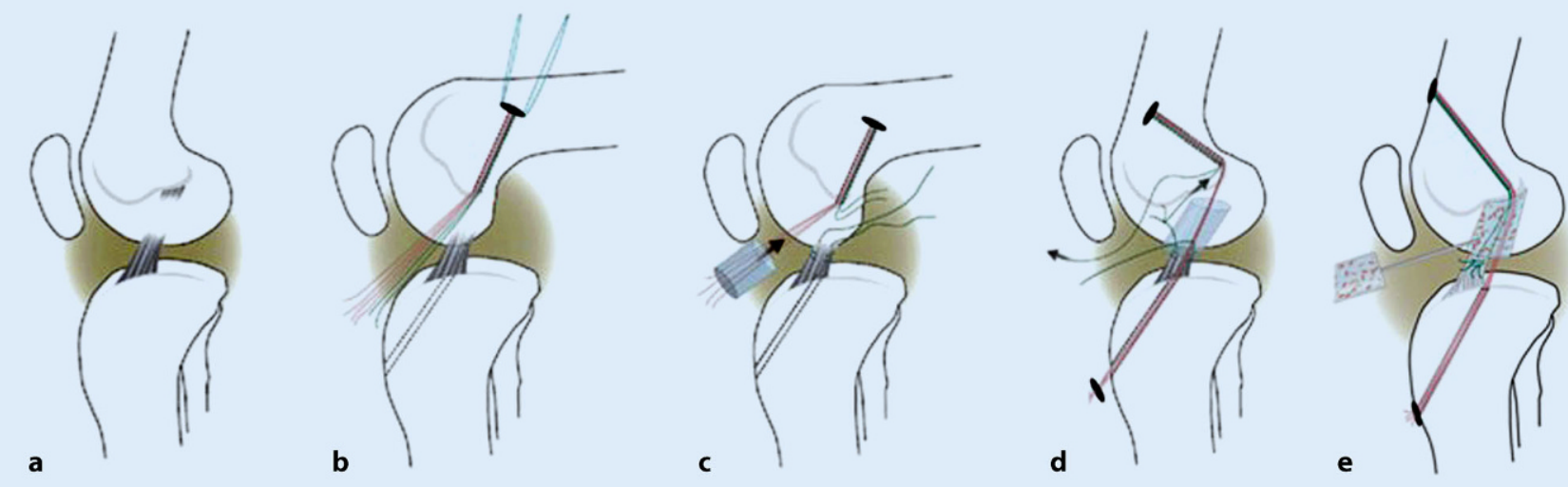

Abb. 6a-e $\Delta$ Schematische Darstellung der Implantation einer Kollagenmatrix zum Schutz der vorderen Kreuzbandfasern vor intraartikulärem plasminreichem Milieu im experimentellen Tiermodell. (Aus [14]). a rupturiertes VKB, b Bohrung eines femoralen und tibialen Tunnels und femorale Ausziehnaht mit Endobuttonfixation, c Kessler Naht des tibialen Stumpfes. Die Kollagenmatrix wird über die femorale Ausziehnaht in intraartikulär eingezogen. $\mathbf{d}$ Die Kollagenmatrix wird in die Notch gezogen und die Ausziehnähte nach tibial ausgeleitet. e Die transtibialen Nähte werden über einen tibialen Button gespannt und verknotet. Die Kollagenmatrix wird mit autologem Blutplasma beimpft

\section{Erhalt des vorderen Kreuz- bandes mittels Refixation und Ligament-Bracing}

Neben der dynamischen Stabilisation des VKB nach Naht wird auch eine nichtdynamische Augmentation von einigen Chirurgen durchgeführt. Hierbei erfolgen die Augmentation und somit interne Stabilisierung des Kniegelenks mittels eines FiberTapes. $\mathrm{Zu}$ dieser Methode gibt es derzeit nur wenige Daten. Eine Arbeitsgruppe verglich die Stabilisierungsergebnisse des Kniegelenks nach alleiniger Naht des VKB-Stumpfes und nach Naht mit Ligament-Bracing mittels FiberTape. Alle diese alternativen Methoden wiesen im experimentellen Modell jedoch geringere Stabilitätswerte auf als nach dynamischer intraligamentärer Stabilisierung mittels Ligamys ${ }^{\circledR}$-Operation. Klinische Ergebnisse sind derzeit nur als 1-Jahres-Ergebnisse von 68 Patienten publiziert worden. Während die klinischen Scoreergebnisse vergleichbar mit Patienten nach VKB Rekonstruktionen waren, so mussten sich jedoch 4 von 68 Patienten in dem 1-Jahres-Zeitraum einer erneuten Operation unterziehen [4]. Mittelfristige Ergebnisse zu diesem Verfahren sind derzeit noch nicht berichtet worden.

\section{Erhalt des vorderen Kreuzbandes und biologische Faktoren}

Die in den vorherigen Abschnitten beschriebenen Techniken zum Erhalt des VKB beschäftigen sich allesamt primär mit der Refixation und möglichst biomechanisch stabilen Redression des tibialen Stumpfes und der Stabilisierung des Kniegelenks.

Ein weiterer wichtiger Problembereich der Heilung des VKB scheinen jedoch die biologischen Heilungsprozesse zu sein. Aufgrund seiner zentralen intraartikulären Lage ist das VKB einem speziellen Gelenkmilieu ausgesetzt, das offensichtlich eine ligamentäre Heilung behindern könnte. Harrold et al. [10] konnten in einer experimentellen Studie zeigen, dass im Gegensatz zu extraartikulär gelegenen Bändern nach VKBRuptur keine Fibrinclot-Bildung an der Stumpfenden festgestellt werden konnte. Als Ursache für die Verhinderung eines bindegewebigen Bridgings wird die hohe Konzentration von Plasmin im Gelenkmilieu angesehen. Plasmin besitzt das Potenzial, sowohl Fibrinverbindungen zu verhindern als auch aufzulösen [3].

An einem potenziellen chirurgischen Lösungsansatz dieser Problematik wird durch die Arbeitsgruppe von Martha Murray gearbeitet. Sie hat bereits eine Kollagenmatrix entwickelt, in die der tibiale Stumpf eingezogen wird und diesen auf diese Weise vor dem intraartikulären Milieu (Plasmin) schützt ([14, 20, 25]; - Abb. 6).

In Tiermodellen konnte die Arbeitsgruppe bereits beweisen, dass die Verwendung der Matrix zu einer verbesserten biologischen Einheilung mit höherer biomechanischer Stabilität und sogar mit verminderter Arthrosebildung führt [25].

\section{Fazit für die Praxis}

- Aus der aktuellen Datenlage lässt sich schließen, dass die Ausheilung der VKB-Ruptur mithilfe moderner Operationstechniken möglich ist.

- Für die isolierte Naht oder Ankerrefixation gibt es derzeit keine ausreichende Studienlage, die Rückschlüsse auf eine reproduzierbare Heilungsquote zulässt. Vergleichbar verhält es sich mit der InternalBracing-Methode mittels FiberTape.

- Die umfangreichste Studienlage gibt es bezüglich des Ligamys ${ }^{\circledR}$-Systems. Die biomechanischen Laborstudien konnten in allen Fällen eine suffiziente und sichere Stabilisierung des Kniegelenks mittels Ligamys ${ }^{\circledR}$ aufzeigen, und auch die klinischen Studien mit Follow-up von 1 bis zu 5 Jahren zeigen vergleichbar gute Scoreergebnisse wie die Rekonstruktionen bei jedoch moderat erhöhter Reruptur- 
quote. Genauere Erkenntnisse über die differenzierte Indikationsstellung und Langzeitergebnisse stehen noch aus.

- Eine interessante und innovative Weiterentwicklung des VKB-Erhaltes würde ein kombinierter biomechanischer und biologischer Ansatz darstellen, der beide Vorgehensweisen vereint.

\section{Korrespondenzadresse}

Prof. Dr. M. Herbort
Klinik für Unfall-, Hand- und
Wiederherstellungschirurgie,
Universitätsklinikum Münster
Albert-Schweitzer-Campus 1,
Gebäude W1, 48149 Münster,
Deutschland
Mirco.Herbort@
uni-muenster.de

\section{Einhaltung ethischer Richtlinien}

Interessenkonflikt. M. Herbort ist Berater bei Medacta, Mathys, Conmed-Linvatec. B. Schliemann und C Kösters sind Berater bei Mathys. J. Glasbrenner, C. Domnick und M.J. Raschke geben an, dass kein Interessenkonflikt besteht.

Dieser Beitrag beinhaltet keine von den Autoren durchgeführten Studien an Menschen oder Tieren.

The supplement containing this article is not sponsored by industry.

\section{Literatur}

1. Achtnich A, Herbst E, Forkel P, Metzlaff $S$, Sprenker F, Imhoff AB, Petersen W (2016) Acute proximal anterior cruciate ligament tears: outcomes after arthroscopic suture anchor repair versus anatomic single-bundle reconstruction. Arthroscopy 32:2562-2569

2. Achtnich A, Rosslenbroich S, Beitzel K, Imhoff $A B$, Petersen W (2017) Arthroscopic refixation of acute proximal anterior cruciate ligament rupture using suture anchors. Oper Orthop Traumatol 29:173-179

3. Andersen RB, Gormsen J (1987) Fibrin dissolution in synovial fluid. Scand J Rheumatol 16:319-333

4. Anthony IC et al (2015) Anterior cruciate ligament repair revisited. Preliminary results of primary repair with internal brace ligament augmentation: a case series. Orthop Muscular Syst 04:188. https:// doi.org/10.4172/2161-0533.1000188

5. Ardern $\mathrm{CL}$, Webster KE, Taylor NF, Feller JA (2011) Return to sport following anterior cruciate ligament reconstruction surgery: a systematic review and meta-analysis of the state of play. $\mathrm{Br} J$ Sports Med 45:596-606

6. DiFelice GS, Villegas C, Taylor S (2015) Anterior cruciate ligament preservation: early results of a novel arthroscopic technique for suture anchor primary anterior cruciate ligament repair. Arthroscopy 31:2162-2171

7. Domnick C, Garcia P, Raschke MJ, Glasbrenner J, Lodde G, Fink C, Herbort M (2017) Trends and incidences of ligament-surgeries and osteotomies of the knee: an analysis of German inpatient records 2005-2013. Arch Orthop Trauma Surg 137:989-995

8. Eggli S, Kohlhof H, Zumstein M, Henle P, Hartel M, Evangelopoulos DS, Bonel H, Kohl S (2015) Dynamic intraligamentary stabilization: novel technique for preserving the ruptured $A C L$. Knee Surg Sports Traumatol Arthrosc 23:1215-1221

9. Häberli J, Henle P, Acklin YP, Zderic I, Gueorguiev B (2016) Knee joint kinematics with dynamic augmentation of primary anterior cruciate ligament repair - a biomechanical study. J Exp Orthop 3:2230. https://doi.org/10.1186/s40634016-0064-2

10. Harrold AJ (1961) The defect of blood coagulation in joints. J Clin Pathol 14:305-308

11. Henle P, Röder C, Perler G, Heitkemper S, Eggli $S$ (2015) Dynamic Intraligamentary Stabilization (DIS) for treatment of acute anterior cruciate ligament ruptures: case series experience of the first three years. BMC Musculoskelet Disord. 16:27

12. Herbort M, Michel P, Raschke MJ, Vogel N, Schulze M, Zoll A, Fink C, Petersen W, Domnick C (2017) Should the Ipsilateral hamstrings be used for anterior cruciate ligament reconstruction in the case of medial collateral ligament insufficiency? Biomechanical investigation regarding dynamic stabilization of the medial compartment by the hamstring muscles. Am J Sports Med 45:819-825. https://doi.org/10.1177/0363546516677728

13. Hoogeslag RAG, Brouwer RW, Veld RHIT, Stephen JM, Amis AA (2017) Improved control of knee laxity after "dynamic" augmentation of ACL suture repai Biomechanical comparison of three $\mathrm{ACL}$ suture repair techniques in the human cadaveric knee. ISAKOSConference, Shanghay.

14. Kiapour AM, Fleming BC, Murray MM (2015) Biomechanical outcomes of bridge-enhanced anterior cruciate ligament repair are influenced by sex in a preclinical model. Clin Orthop Relat Res 473:2599-2608

15. Kösters C, Herbort M, Schliemann B, Raschke MJ, Lenschow S (2015) Dynamic intraligamentary stabilization of the anterior cruciate ligament: operative technique and short-term clinical results. Unfallchirurg Springer Berlin Heidelberg 118:364-371

16. van der List JP, DiFelice GS (2017) Preoperative magnetic resonance imaging predicts eligibility for arthroscopic primary anterior cruciateligament repair. Knee Surg Sports Traumatol Arthrosc 32:2562

17. van der List JP, DiFelice GS (2017) Primary repair of the anterior cruciate ligament: a paradigm shift. Surgeon 15:161-168

18. Lubowitz JH (2014) Anatomic ACL reconstruction produces greater graft length change during knee range-of-motion than transtibial technique. Knee Surg 22:1190-1195

19. Marshall JL, Warren RF, Wickiewicz TL, Reider B (1979) The anterior cruciate ligament: a technique of repair and reconstruction. Clin Orthop Relat Res 143:97-106

20. Murray MM, Fleming BC (2013) Use of a bioactive scaffold to stimulate anterior cruciate ligament healing also minimizes posttraumatic osteoarthritis after surgery. Am J Sports Med 41:1762-1770
21. Paessler HH, Deneke J, Dahners LE (1992) Augmented repair and early mobilization of acute anterior cruciate ligament injuries. Am J Sports Med 20:667-674

22. Schliemann B, Lenschow S, Domnick $C$, Herbort $M$, Häberli J, Schulze M, Wähnert D, Raschke MJ, Kösters C (2015) Knee joint kinematics after dynamic intraligamentary stabilization: cadaveric study on a novel anterior cruciate ligament repair technique. Knee Surg 25:1-7

23. Schliemann B, Glasbrenner J, Rosenbaum D, Lammers K, Herbort M, Domnick C, Raschke MJ, Kösters C (2017) Changes in gait pattern and early functional results after $A C L$ repair are comparable to those of $A C L$ reconstruction. Knee Surg Sports Traumatol Arthrosc 43:121

24. Schliemann B, Herbort M, Lenschow S, Raschke MJ, Kösters C (2015) Dynamische intraligamentäre Stabilisierung frischer Rupturen des vorderen Kreuzbandes (Ligamys). Trauma Berufskrankh Springer Berlin Heidelberg 18:254-259

25. Vavken P, Fleming BC, Mastrangelo AN, Machan JT, Murray MM (2012) Biomechanical outcomes after bioenhanced anterior cruciate ligament repair and anterior cruciate ligament reconstruction are equal in a porcine model. Arthroscopy 28:672-680

26. Weaver JK, Derkash RS, Freeman JR, Kirk RE, Oden RR, Matyas J (1985) Primary knee ligament repair-revisited. Clin Orthop Relat Res 199:185-191 